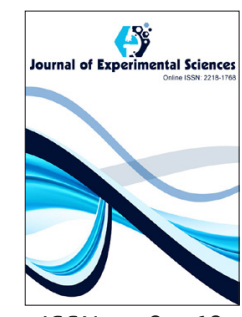

ISSN: $2218-1768$
Received: March 19, 2021

Revised: July 18, 2021

Accepted: July 20, 2021

Published: August 10, 2021

*Corresponding Author:

Vladimir A. Smirnov,

E-mail: vla_smirnov@mail.ru

\section{Swim test for joint angles dispersion analysis during hind limb motor function assessment in SCI models}

\author{
Vladimir A. Smirnovi*, Sergey A. Bazanovich², Mikhail Ya. Yadgarov², \\ Marina A. Zvyagintseva², Andrew A. Grin", Sergey I. Ryabov"
}

'Department of Neurosurgery, N.V. Sklifosovsky Research Institute of Emergency Care, Moscow, Russian Federation, ${ }^{2}$ Laboratory of Stem Cells, National Medical Research Center of Cardiology, Moscow, Russian Federation

\begin{abstract}
In the most of functional studies, various stress tests are used to assess functional improvement following spinal cord injury in animal models. However, available methods of motor function evaluation are not always accurate and unbiased. The main objective of the study was to create a new method of motor activity assessment in minor animal models of spinal cord injury. This method should provide objective and accurate evaluation of limb motor function in models having severe neurological disorders following contusion spinal cord injury. Swimming test was used as the key behavioral test. To assess the motor function of swimming animals' hind limbs, we measured angles of hind limbs movements adjusted to motion direction axis. Then we calculated individual angles dispersion for each joint and limb using the parameters of angles sample dispersion and amplitude-depending dispersion. Current study included two groups of Sprague-Dawley rats: control group and a group of animals having moderate thoracic spinal cord contusion injury. Control animals demonstrated stable dispersion indicators for 6 weeks of follow-up. In the experimental group, a tendency to improvement of motor function in hind limbs between 1 and 3 weeks was revealed followed by stabilization and preservation of both indicators between 3 and 6 weeks. Provided method based on the measurement of joint angles adjusted to the movement direction axis followed by calculation of indicators of variance of a random variable and amplitude-depending variance can be effective and objective alternative for motor function evaluation.
\end{abstract}

KEYWORDS: Swim test, joint angles, dispersion, spinal cord injury, animal models, functional improvement

\section{BACKGROUND}

Current techniques for hind limb motor function assessment in minor animal models include multiple behavioral tests, such as open-field test (Basso, 1995; Bunge, 2008), beam-walking test (Hicks et al., 1975; von Euler et al., 1996; Cummings et al., 2007) swim test (Gullo et al., 2008), rotarod device (Dunham \& Miya, 1957) and others (Mever et al., 1979; Gale et al., 1985; Panjabi, et al., 1998; Muir \& Webb, 2000; Ahmed et al., 2019; Chao et al., 2012).

Most behavioral tests (e.g., beam-walking test and Rotarod) demonstrate highly limited sensitivity and/or specificity and often provide imprecise results in animals having severe motor dysfunction (Barros Filho \& Molina, 2008; Zörner et al., 2010). Moreover, some tests are inapplicable for such animal models resulting in complete absence of any significant values. For instance, rats with severe and even moderate contusion spinal cord injury (SCI) (Basso, 1995) are unable to perform Rotarod test even in several months post-injury. Considering complexity and impreciseness of the most behavioral tests application in animal models of severe SCI swim test seems to be promising in many aspects. First, water-holding effect allows performing swim test and assessing motor function even in animals having severe neurological deficit (Bejan \& Marden, 2006). Second, water itself stimulates continuous limb movements in all swimming animals, otherwise rats begin to sink (Gruner \& Altman, 1980; Baffour et al., 1995). Third, swimming is one of the quickest subtypes of the main motor pattern involving high extensor activity reaching their peak contraction (Dittgen et al., 2012). Fourth, swimming process results in additional limb elongation due to the absence of gravitational effect (Gullo et al., 2008; Magnuson et al., 2009). This allows animal models to achieve maximal amplitude action in all joints (Gruner \& Altman, 1980). These factors result in more significant and precise assessment of motor function and promote efficient examination of animals having subtotal motor neurological deficit (Sedý et al., 2008).

Copyright: (-) The authors. This article is open access and licensed under the terms of the Creative Commons Attribution License (http://creativecommons.org/licenses/by/4.0/) which permits unrestricted, use, distribution and reproduction in any medium, or format for any purpose, even commercially provided the work is properly cited. Attribution - You must give appropriate credit, provide a link to the license, and indicate if changes were made. 
The kinematics of rat movements during swimming process are widely and well explored. Healthy animals keep their bodies at a slight angle to the water surface during swimming. Only $30 \%$ of their head, neck and body are kept under the water. Animal's tail is usually held on the water surface or right under it. Generally, forward motion is achieved by active cyclic movement of hind limbs. Front limbs are usually held under the head. Moreover, rats do not use front limbs in swimming process, but occasionally can involve them for orientation and direction correction. A relative body rotation degree to movement direction axis is usually insignificant (Smith et al., 2006).

Swimming process in animal models of spinal cord injury is completely different. Animals having significant deficit of hind limbs motor function demonstrate considerable involvement of front limbs to the swimming process (Smith et al., 2006). Hind limbs are also involved depending on the severity of motor dysfunction. SCI models also demonstrate body instability and excessive submergence under the water surface during swimming (Smith et al., 2006a; Smith et al., 2006b).

In the most current preclinical studies motor function assessment is performed using 17-point Louisville swim scale (Gruner \& Altman, 1980; Xu et al., 2015; Sitoci-Ficici et al.,2018; Smirnov et al., 2019) developed by the group of David Magnuson in 2006 (Smith et al., 2006a). This scale estimates 3 main parameters of swimming process: (i) front limbs movements, (ii) hind limbs movements and (iii) body positioning. Each parameter is estimated using a certain number of points. A total value can range between 0 (the most severe SCI) and 17 (normal) points. Level between 0 and 5 points corresponds to severe neurological deficit including significant motor dysfunction and/or inability to swim. Value ranging between 6 and 11 points indicates moderate neurological deficit (e.g. ability to perform swimming action is limited). Value between 12 and 17 points corresponds to minimal deficit or normal level of motor function (Xu et al., 2015).

Such technique of motor function assessment is significantly restricted. Despite high sensitivity of the swimming test, the preciseness of the conducted analysis is much lower in animals demonstrating severe motor dysfunction (severe paraparesis and/or paraplegia) (Smith et al., 2006b). Active involvement of front limbs in swimming process and inability to use hind limbs results in the absence of any changes of body stability and angle of body rotation. Consequently, dynamics of these parameters have no effect on the final result. Moreover, this method evaluates animal's general swimming potential and misses the examination of each individual parameter involved in swimming process and reflecting limb motor function. Finally, Louisville scale is based on highly subjective point assessment demonstrating the hind limb motor function very roughly (Xu et al., 2015; Smith et al., 2006a).

For this reason, we developed a certain technique based on swimming test for precise and objective hind limb motor function evaluation in small laboratory animals demonstrating significantly higher level of preciseness and objectivity comparing to other currently used tests.

\section{MATERIALS AND METHODS}

\section{Swimming Test}

We performed one of the most recent modifications of swim test developed by the group of Martin Schwab in the early 2000s (Liebscher et al., 2005). Animals were placed into a transparent rectangular swimming pool (size parameters $-150 \times 40 \times 13$ $\mathrm{cm})$ filled with room temperature water $\left(23-25^{\circ} \mathrm{C}\right)$. Water level was high enough to prevent animals from touching the bottom. Track-guide for low-motion camera was placed in front of the pool to record all tests for the subsequent additional and/or computed analysis (Figure 1).

Animals were marked with 5 dots each using permanent black marker prior to testing (Figure 2), each mark indicated a certain joint of hind limb: \# 1 mark - distal part of metatarsus or back side of the foot; \#2 mark - ankle joint; \#3 mark - knee joint; \#4 mark - hip joint; \#5 mark - sacroiliac joint. Marks \# 1 and \#2 are used for measurement of ankle joint angle adjusted

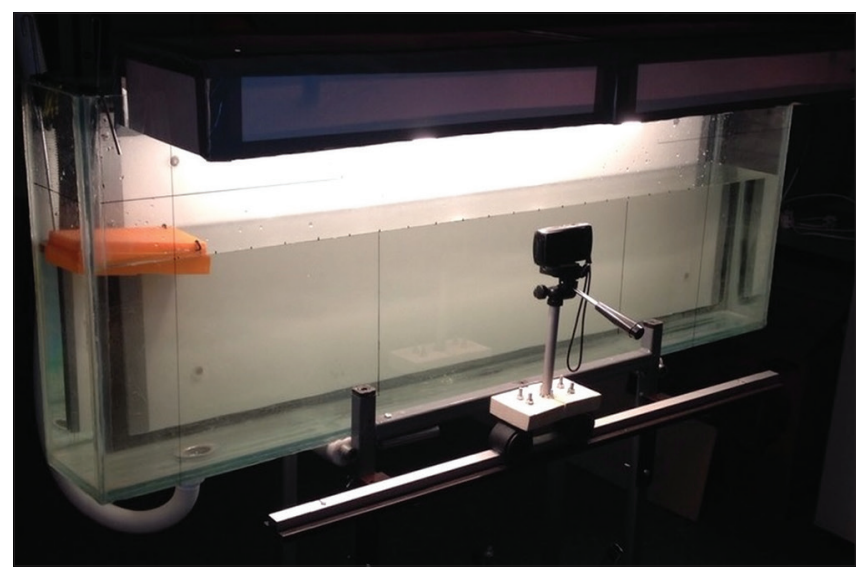

Figure 1: Swimming pool in modification by Martin Schwab et al. A rectangular swimming pool $(150 \times 40 \times 13 \mathrm{~cm})$ filled with room temperature water was used. Additional video record of swim tests was performed using low-motion camera

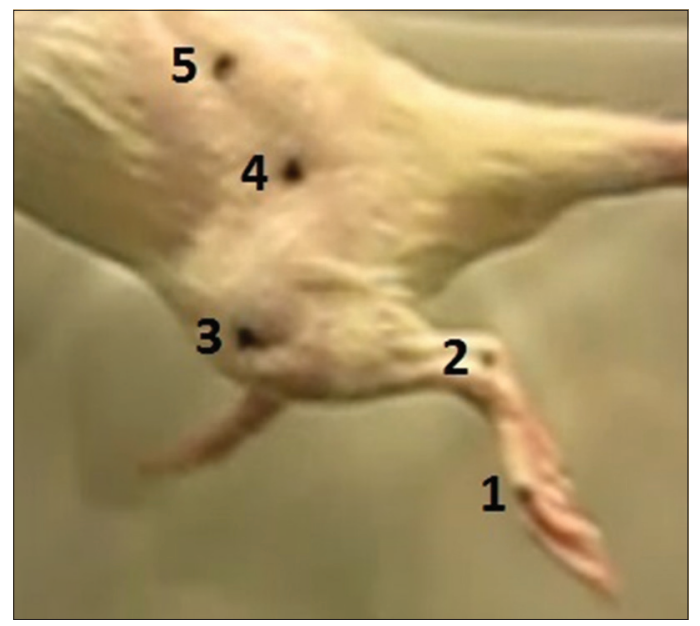

Figure 2: Position of hind limb joint marks. Each mark corresponds to a certain joint: \#1 - distal part of metatarsus or back side of the foot; \#2 - ankle joint; \#3 - knee joint; \#4 - hip joint; \#5 - sacroiliac joint 
to the motion direction axis. Marks \#2 and \#3 enable the measurement of knee joint angle adjusted to the motion direction axis. Marks \#3 and \#4 are used for measurement of hip joint angle adjusted to the motion direction axis. Mark \#5 is an auxiliary mark used for angle line formation control. Such schematic representation facilitates the analysis of motor function in the corresponding joints during examination and allows performing computer analysis of video records later (Figure 3b).

Swim tests were recorded using low-motion camera. Video records were divided into single shots and used for measurement of joint angles adjusted to the to the motion direction axis for each individual joint during each rowing cycle. Then dispersion calculation was performed for each joint using one of two described above techniques. A cumulative motor function of the whole limb was evaluated using all values of three limb joints.

\section{Measurement of Joint Angles}

There are sparse studies evaluating hind limb motor function in small laboratory animals using swim test including not only the 17-point Louisville swim scale, but also the measurement of hind limbs joint angles (Johnston \& Bekoff, 1992; Magnuson et al., 2009; Zörner et al., 2010). The technique of joint angle measurement is supposed to be more precise objective comparing to Louisville swim scale due to almost complete absence of subjective analysis performed by the investigator. The assessment of hind limb motor function during swim test includes the measurement of hip, knee and ankle joints angles. Each angle is formed using connection of skin marks placed on the limbs surface prior to the examination. Hip joint angle is formed by the marks of sacroiliac, hip and knee joints and corresponding lines (sacroiliac/hip - hip/knee). Knee joint angle is formed by the marks of hip, knee and ankle joints (hip/ knee - knee/ankle). Ankle joint angle is formed by the marks of knee and ankle joints and distal part of metatarsus (knee/ ankle - ankle/metatarsus) (Figure 3a).

According to the laws of conventional mechanics, changes of spatial position of the animal body and its parts rely on the system of coordinates related to the body (Goldstein et al.,

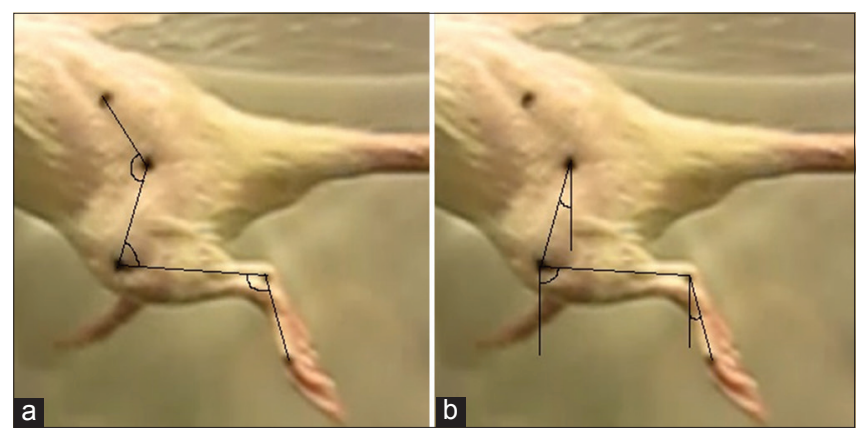

Figure 3: Schematic representation of two types of joint angles measurement. Advantages and disadvantages as well as limitations are provided in the text. (a) Joint angles without correctly introduced reference system of coordinates. (b) Joint angles adjusted to the motion direction axis
2002). Such system of coordinates (reference system) allows to set the baseline position of the body. Each analysis of body motion should initially specify the implemented system of coordinates resulting in limitation of analyzed movements patterns (Goldstein et al., 2002). All current studies using swimming test for articular angles assessment and hind limb motor function evaluation in minor animal models did not have an appropriate reference system (Sedý et al., 2008; Magnuson et al., 2009; Xu et al., 2015; Sitoci-Ficici et al., 2018). Joint movements analysis was performed without adjustment both to motion direction axis and animal body position. In this case, mentioned method of joint angles measurement is inapplicable for motion description. Thus, we developed a new technique of hind limb motor function assessment using a different method of joint angles measurement adjusted to motion direction axis during swim test performance (Figure 3b). Such technique of joint angle measurement allows performing the correct analysis of hind limb movement pattern. Moreover, it enables assessing other parameters of motion, such as limb movement rate, strength level and precise kinematics of limb movements.

\section{Sample Dispersion}

Dispersion parameter is an indicator of random variable variation spread comparing to its mathematical expectation (Glantz, 1998). It provides highly significant evaluation of the joint angles' distribution, adjusted to motion direction axis for each individual joint at each individual motion cycle (these values are considered to be equally distributed random variables). It also allows estimating the deviation of the measured angle values comparing to its mathematical expectation.

Joint angle dispersion can be calculated using the following formula:

$$
D[x]=\frac{\sum_{i=1}^{n}\left(x_{i}-\bar{x}\right)^{2}}{n-1}
$$

$x_{i}$ - specific value of individual joint flexion angle;

$\bar{x}$-mean value of several individual joint flexion angles;

$n-$ number of measured angles for each joint.

Dispersion parameter is calculated for a series of flexion angles measured for each joint and adjusted to the motion direction axis. It allows calculating the numeric value of rotation degree in each individual joint and also estimating the cumulative value for the whole limb in each individual rowing cycle.

\section{Angle Amplitude-dependent Dispersion}

A series of hind limbs motor function kinematics examinations based on the measured joint angles' values adjusted to the motion direction axis during swim test revealed that dynamics of hind limbs joint angle values resembles the standard function $y=\sin x$ (Figure 4).

Thus, the structure of joint angle dynamics is close to harmonic oscillations described by the following formula (1): 


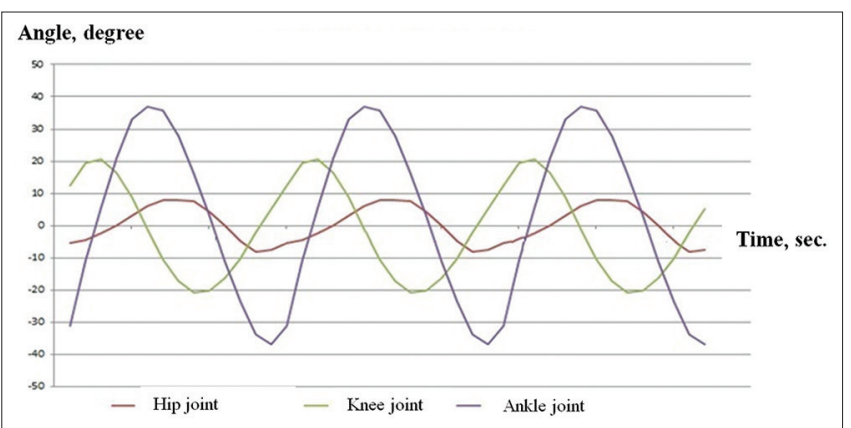

Figure 4: Dynamics chart of joint angle values measured in the swim test

$$
x=A \sin (\omega t)
$$

$\mathrm{x}$ - shift;

A - movement amplitude;

$\omega t-$ cyclic frequency.

Since animal's limb movements during swimming process are continuous, the value of angle variations also changes continuously. Then dispersion of the continuous value is described by another formula (2):

$$
D[x]=\int_{-\infty}^{+\infty}(x-M[x])^{2} f(x) d x
$$

$\mathrm{D}[\mathrm{x}]$ - dispersion of the random value;

$\mathrm{M}[\mathrm{x}]$ - mathematical expectation;

$f(x)$ - density of the random value probability.

In this context, the density of the distribution probability $f(x)$ of the function ( 1 ) is calculated using the following formula (3):

$$
f(x)=\frac{1}{\pi \sqrt{A^{2}-a^{2}}}
$$

Transfer of the calculated density of distribution probability into formula (2) (while $\mathrm{M}[\mathrm{x}]=0$ for harmonic oscillations) results in the following formula (4):

$$
D[x]=\int_{-A}^{A} \frac{x^{2}}{\pi \sqrt{A^{2}-a^{2}}}
$$

Calculating the integral within the single movement cycle using formula (5):

$$
D[x]=\int_{-\frac{\pi}{2}}^{\frac{\pi}{2}} \frac{x^{2}}{\pi \sqrt{A^{2}-a^{2}}}
$$

Finally, dispersion calculation can be performed:

$$
D=\frac{A^{2}}{2}
$$

$\mathrm{A}$ - amplitude of the movement cycle.

The obtained formula (6) resembles the formula of dispersion for joint flexion angles. However, the one should specify a value of the maximal angle amplitude for each individual joint. Moreover, this formula reflects the physical significance of dispersion parameter.

\section{RESULTS}

First group of minor laboratory animals included 10 healthy female Sprague-Dawley rats (weight range between 250 and $350 \mathrm{~g})$ studied in a series of swim tests within 6-week period with l-week interval. Two alternative values of dispersion were calculated using both techniques of parameter assessment. Cumulative amplitude-dependent dispersion dynamics considering measurements of all three limb joints are provided in Table 1. Dynamics of selective angle dispersion adjusted to the motion direction axis and considering measurements of all three limb joints are provided in Table 2. Interestingly, the value of dispersion demonstrated significant stability within 6-week observational period. Moreover, the value of amplitudedependent dispersion is insignificantly different from the value of cumulative dispersion.

Second group of animals included 14 female Sprague-Dawley rats (weight range between 250 and $350 \mathrm{~g}$ ) having moderate contusion spinal cord injury at the level of Th9 vertebra using standard "weight-drop" technique (Basso et al., 1996). These SCI models were tested weekly for 6 weeks postoperative (p/o) with l-week interval. Similarly, two alternative values of dispersion were calculated using both techniques of parameter assessment. Cumulative amplitude-dependent dispersion dynamics considering measurements of all three limb joints are provided in Table 3. Dynamics of selective angle dispersion adjusted to the motion direction axis and considering measurements of all three limb joints are provided in Table 4. We revealed a clear tendency to increase of hind limbs motor function between $1^{\text {st }}$ and $3^{\text {rd }}$ weeks $\mathrm{p} / \mathrm{o}$ and preservation of the reached level between $3^{\text {rd }}$ and $6^{\text {th }}$ weeks $\mathrm{p} / \mathrm{o}$. At the same time, the value of amplitude-dependent dispersion is also insignificantly different from the value of cumulative dispersion.

Table 1: Cumulative dispersion dynamics depending on the movement amplitude and considering measurements of all three limb joints in the group of healthy animals $(n=10)$

Swim test timing 1 week 2 weeks 3 weeks 4 weeks 5 weeks 6 weeks

Amplitude-

dependent

dispersion

degree $^{2}$

Table 2: Dynamics of selective angle dispersion adjusted to the motion direction axis and considering measurements of all three limb joints in the group of healthy animals $(n=10)$

Swim test timing 1 week 2 weeks 3 weeks 4 weeks 5 weeks 6 weeks

Selective angle $2763,342768,762762,212760,892771,852766,22$

dispersion

adjusted to the

motion axis,

degree $^{2}$ 
Table 3: Cumulative amplitude-dependent dispersion dynamics considering measurements of all three limb joints in the group of SCI models $(n=14)$

Swim test timing 1 week 2 weeks 3 weeks 4 weeks 5 weeks 6 weeks

Amplitude- $\quad 334,651047,151206,541123,361165,07 \quad 1090,89$

dependent

dispersion

degree $^{2}$

Table 4: Dynamics of selective angle dispersion adjusted to the motion direction axis and considering measurements of all three limb joints in the group of SCI models $(n=14)$

Swim test timing 1 week 2 weeks 3 weeks 4 weeks 5 weeks 6 weeks
$\begin{aligned} & \text { Selective angle } 335,53 \quad 1049,29 \\ & \text { dispersion adjusted }\end{aligned}$
to the motion axis,
degree $^{2}$

\section{DISCUSSION}

Swimming test demonstrates several important advantages comparing to other behavioral tests widely used for motor function evaluation in minor animal models. Currently used techniques for swim test results evaluation are restricted to Louisville swim scale. This scale has proved to be effective and objective in multiple animal models of various diseases and injuries. Moreover, it covers motor potential of the whole animal's body. However, detailed examination of this scale and a method of kinematic evaluation resulted in a number of limitations. Louisville scale demonstrated multiple disadvantages preventing from precise and objective analysis in animals having severe neurological deficit resulting from spinal cord injury.

While swimming, healthy rats use only the hind limbs for moving and keep front limbs under the head. According to Louisville swim scale active involvement of front limbs results in 0 points. Importantly, even slight motor deficit in hind limbs (e.g. in animals with all types of SCI) always results in active involvement of front limbs in swimming cycle. So, such swimming pattern is obviously inapplicable for animal models of SCI. Otherwise, each analysis results in serious understatement of scale values, even in animals demonstrating motor improvement following SCI of any severity.

Another parameter assessed by Louisville swim scale is the degree of body inclination from the water surface. Healthy animals keep their bodies under a slight angle from the surface while swimming. Neurological deficit results in body immersion under the water surface and increase of body-surface angle. Moreover, the increase of deficit severity results in larger bodysurface angle. However, almost all SCI models demonstrate significant body immersion regardless the severity of original injury and even in late post-SCI period. This is the second reason for such pattern to understate the final motor value in animals having neurological deficit following SCI.

A similar situation is observed concerning body rotation degree in animal models of SCI. Healthy animals demonstrate an insignificant body rotation degree while swimming. On the other hand, significant increase of body rotation degree is observed following SCI of any severity. Again, this parameter results in understatement of final value of Louisville scale.

We suppose, that front limbs motor activity, body-surface angle value and body rotation degree are the indirect parameters and do not describe motor potential completely both in healthy animals and after SCI of any severity. Obviously, not enough attention is paid to motor activity of hind limbs, which is the key factor of motor function improvement following SCI. Despite relatively high sensitivity of the test, analysis reliability appears to be much lower in animals having severe motor dysfunction (severe paraparesis or paraplegia). Another disadvantage includes the subjectivity of scale analysis highly dependent from investigator's opinion and describing swimming kinematics insufficiently.

Since hind limbs movements are the key physiological parameter of motor function improvement and dynamics of general neurological deficit, it should be addressed and analyzed. Measuring of hind limbs joint angles during different phases of rowing cycle provides both an objective evaluation of each individual joint rotation degree and detailed description of the whole limb movement. Again, main disadvantage of the currently used methods for joint angles measurement is the absence of an appropriate reference system resulting in inability to describe and analyze the hind limbs movements in animal models of SCI. Therefore, we developed a technique of joint angles measurement adjusted to general motion direction axis free of mentioned disadvantages of previously described methods.

Importantly, the range of measured joint angles adjusted to motion direction axis is rather narrow. This doesn't allow to reliably compare motor function values in animals having different levels of neurological deficit at one time point and reveal significant differences between them. This requires a new parameter allowing to evaluate differences variations of each joint angle adjusted to motion direction axis.

A selective dispersion is a general parameter focused on reliable assessment of variations range of joint angles adjusted to the motion direction axis for each individual joint. Sensitivity of selective dispersion is higher due to squared value of numerator and demonstrates wider range of values comparing to absolute values of the same joint angles. However, selective dispersion is the statistical parameter providing, in our case, the descriptive criteria for axis-adjusted joint angles selection. For this reason, we have to define the physical meaning of dispersion parameter considering the kinematic structure of each joint movement.

Amplitude-dependent dispersion parameter reflects the physical meaning of selective dispersion. It is based on the dynamics of joint angles changes of hind limbs in swimming animals and is calculated using key physical principles of oscillatory motions. According to our results, both parameters of selective dispersion and amplitude-dependent dispersion are close in their values and can be used for description and analysis of hind limbs motor 
function in minor animal models, both healthy and having neurological deficit of any severity.

Provided technique is based on the calculation of evaluative parameters and comprises several steps: i) marking of animal's hind limbs with five contrast dots; ii) registration of swimming process using slow-motion video camera; iii) storyboarding of obtained video files; iv) measurement of joint angles adjusted to the motion direction axis in three main limb joints for each individual rowing cycle; v) calculation of selective sample dispersion and angle amplitude-dependent dispersion parameters for the whole limb and separately for each joint for each individual rowing cycle. Since we use only the calculated parameters to assess motor function of hind limbs, provided technique is more objective and precise comparing both to Louisville swim scale and other current assessment methods.

\section{CONCLUSION}

Performed analysis demonstrated that measurement of joint angles adjusted to the motion direction axis followed by calculation of sample selective dispersion and angle amplitudedependent dispersion for these angles are valuable, precise and efficient methods of hind limbs motor function evaluation in both healthy animal models and animals having neurological deficit of various severity.

\section{Highlights}

- Current behavioral tests are restricted in their sensitivity if are applied to models having severe neurological deficit;

- Swimming test allows to effectively assess motor function even in animals with severe deficit;

- Currently used Louisville swim scale does not reflect dynamics of hind limbs motor function following SCI;

- Joint angles analysis is more sufficient than Louisville swim scale, but absolute values also do not reflect motor function improvement;

- Sample dispersion is a much more sensitive parameter demonstrating even slight dynamics of motor function;

- Amplitude-dependent dispersion reflects the physical meaning of sample dispersion and can be used as criterion of motor function improvement.

\section{AUTHOR CONTRIBUTIONS}

Vladimir A. Smirnov: conceptualization, investigation, supervision, validation, writing - original draft, writing - review \& editing. Sergey Bazanovich: conceptualization, data curation, investigation, methodology, software, validation, writing original draft. Mikhail Yadgarov: conceptualization, data curation, investigation, methodology, visualization, writing original draft. Marina Zvyagintseva: investigation, visualization. Andrew Grin': conceptualization, supervision, formal analysis, resourses. Sergey Ryabov: conceptualization, methodology, supervision, writing - review \& editing. Vladimir N. Smirnov: conceptualization, supervision, formal analysis, funding acquisition, project administration, writing - review \& editing.

\section{REFERENCES}

Ahmed, R. U., Alam, M., \& Zheng, Y. P. (2019). Experimental spinal cord injury and behavioral tests in laboratory rats. Heliyon, 5(3), e01324. https://doi.org/10.1016/j.heliyon.2019.e01324

Baffour, R., Achanta, K., Kaufman, J., Berman, J., Garb, J. L., Rhee, S., \& Friedmann, P. (1995). Synergistic effect of basic fibroblast growth factor and methylprednisolone on neurological function after experimental spinal cord injury. Journal of Neurosurgery, 83(1), 105-110. https://doi.org/10.3171/jns.1995.83.1.0105

Barros Filho, T. E., \& Molina, A. E. (2008). Analysis of the sensitivity and reproducibility of the Basso, Beattie, Bresnahan (BBB) scale in Wistar rats. Clinics, 63(1), 103-108. https://doi.org/10.1590/s180759322008000100018

Basso, D. M., Beattie, M. S., \& Bresnahan, J. C. (1995). A sensitive and reliable locomotor rating scale for open field testing in rats. Journal of Neurotrauma, 12(1), 1-21. https://doi.org/10.1089/neu.1995.12.1

Basso, D. M., Beattie, M. S., \& Bresnahan, J. C. (1996). Graded histological and locomotor outcomes after spinal cord contusion using the NYU weight-drop device versus transection. Experimental Neurology, 139(2), 244-256. https://doi.org/10.1006/exnr.1996.0098

Bejan A., Marden J. H. (2006). Constructing animal locomotion from new thermadynamics theory: Although running, flying and swimming appear to be distinctly different types of movement, they may have underlying physics in common. American Scientist, 94(4), 342-349. https://www.jstor.org/stable/27858803

Bunge M. B. (2008). Novel combination strategies to repair the injured mammalian spinal cord. Journal of Spinal Cord Medicine, 31(3), 262-269. https://doi.org/10.1080/10790268.2008.11760720

Chao, O. Y., Pum, M. E., Li, J. S., \& Huston, J. P. (2012). The grid-walking test: assessment of sensorimotor deficits after moderate or severe dopamine depletion by 6-hydroxydopamine lesions in the dorsal striatum and medial forebrain bundle. Neuroscience, 202, 318-325. https://doi.org/10.1016/j.neuroscience.2011.11.016

Cummings, B. J., Engesser-Cesar, C., Cadena, G., \& Anderson, A. J. (2007). Adaptation of a ladder beam walking task to assess locomotor recovery in mice following spinal cord injury. Behavioural Brain Research, 177(2), 232-241. https://doi.org/10.1016/j.bbr.2006.11.042

Dittgen, T., Pitzer, C., Plaas, C., Kirsch, F., Vogt, G., Laage, R., \& Schneider, A. (2012). Granulocyte-colony stimulating factor (G-CSF) improves motor recovery in the rat impactor model for spinal cord injury. PLoS One, 7(1), e29880.

https://doi.org/10.1371/journal.pone.0029880

Dunham, N. W., \& Miya, T. S. (1957). A note on a simple apparatus for detecting neurological deficit in rats and mice. Journal of the American Pharmaceutical Association. American Pharmaceutical Association, 46(3), 208-209. https://doi.org/10.1002/jps.3030460322

Gale, K., Kerasidis, H., \& Wrathall, J. R. (1985). Spinal cord contusion in the rat: behavioral analysis of functional neurologic impairment. Experimenta/Neurology, 88(1), 123-134. https://doi.org/10.1016/00144886(85)90118-9

Glantz, S. A. (1998). Primer of Biostatistics. McGraw-Hill.

Goldstein, H., Poole C., \& Safko J. (2002). Classical mechanics. USA Addison-Wesley.

Gruner, J. A., \& Altman, J. (1980). Swimming in the rat: analysis of locomotor performance in comparison to stepping. Experimental Brain Research, 40(4), 374-382. https://doi.org/10.1007/BF00236146

Gullo, M., Hochreutener, E., Schnell, D., Scholl, J., Schwab, M. E., \& Schnell, L. (2008). The Schnell Swim Test (SST) to measure motor function and recovery in spinal cord injured rats. Proceedings of Measuring Behavior, pp 35-36.

Hicks, S. P., \& D'Amato, C. J. (1975). Motor-sensory cortex-corticospinal system and developing locomotion and placing in rats. American Journal of Anatomy, 143(1), 1-42. https://doi.org/10.1002/ aja.1001430102

Johnston, R. M., \& Bekoff, A. (1992). Constrained and flexible features of rhythmical hindlimb movements in chicks: kinematic profiles of walking, swimming and airstepping. Journal of Experimental Biology, $171,43-66$.

Liebscher, T., Schnell, L., Schnell, D., Scholl, J., Schneider, R., Gullo, M., Fouad, K., Mir, A., Rausch, M., Kindler, D., Hamers, F. P., \& Schwab, M. E. (2005). Nogo-A antibody improves regeneration and locomotion of spinal cord-injured rats. Annals of neurology, 58(5), 706-719. https:// doi.org/10.1002/ana.20627 
Magnuson, D. S., Smith, R. R., Brown, E. H., Enzmann, G., Angeli, C., Quesada, P. M., \& Burke, D. (2009). Swimming as a model of taskspecific locomotor retraining after spinal cord injury in the rat. Neurorehabilitation and Neural Repair, 23(6), 535-545. https://doi. org/10.1177/1545968308331147

Meyer, O. A., Tilson, H. A., Byrd, W. C., \& Riley, M. T. (1979). A method for the routine assessment of fore- and hindlimb grip strength of rats and mice. Neurobehavioral Toxicology, 1(3), 233-236.

Muir, G. D., \& Webb, A. A. (2000). Assessment of behavioural recovery following spinal cord injury in rats. European Journal of Neuroscience, 12(9), 3079-3086. https://doi.org/10.1046/j.1460-9568.2000.00205.x

Panjabi, M. M., \& Wrathall, J. R. (1988). Biomechanical analysis of experimental spinal cord injury and functional loss. Spine, 13(12), 1365-1370. https://doi.org/10.1097/00007632-198812000-00007

Sedý, J., Urdzíková, L., Jendelová, P., \& Syková, E. (2008). Methods for behavioral testing of spinal cord injured rats. Neuroscience and Biobehavioral Reviews, 32(3), 550-580. https://doi.org/10.1016/j. neubiorev.2007.10.001

Sitoci-Ficici, K. H., Matyash, M., Uckermann, O., Galli, R., Leipnitz, E., Later, R., Ikonomidou, C., Gelinsky, M., Schackert, G., \& Kirsch, M. (2018). Non-functionalized soft alginate hydrogel promotes locomotor recovery after spinal cord injury in a rat hemimyelonectomy model. Acta Neurochirurgica, 160(3), 449-457. https://doi.org/10.1007/ s00701-017-3389-4

Smirnov, V. A., Yadgarov, M. Y., Bazanovich, S. A., Grin, A. A., Krylov, V. V., Ryabov, S. I. et al. (2019). Improvement of hind limbs motor function evaluation following systemic cord blood stem cell therapy using swimming test computed analysis. In genetics, genetic disorders and stem cell (pp. 82-82).

Smith, R. R., Burke, D. A., Baldini, A. D., Shum-Siu, A., Baltzley, R., Bunger, M., \& Magnuson, D. S. (2006a). The Louisville Swim Scale: a novel assessment of hindlimb function following spinal cord injury in adult rats. Journal of Neurotrauma, 23(11), 1654-1670. https://doi. org/10.1089/neu.2006.23.1654

Smith, R. R., Shum-Siu, A., Baltzley, R., Bunger, M., Baldini, A., Burke, D. A., \& Magnuson, D. S. (2006b). Effects of swimming on functional recovery after incomplete spinal cord injury in rats. Journal of Neurotrauma, 23(6), 908-919. https://doi.org/10.1089/neu.2006.23.908

von Euler, M., Akesson, E., Samuelsson, E. B., Seiger, A., \& Sundstrom, E. (1996). Motor performance score: a new algorithm for accurate behavioral testing of spinal cord injury in rats. Experimental neurology, 137(2), 242-254. https://doi.org/10.1006/exnr.1996.0023

Xu, N., Åkesson, E., Holmberg, L., \& Sundström, E. (2015). A sensitive and reliable test instrument to assess swimming in rats with spinal cord injury. Behavioural Brain Research, 291, 172-183. https://doi. org/10.1016/j.bbr.2015.05.004

Zörner, B., Filli, L., Starkey, M. L., Gonzenbach, R., Kasper, H., Röthlisberger, M., Bolliger, M., \& Schwab, M. E. (2010). Profiling locomotor recovery: comprehensive quantification of impairments after CNS damage in rodents. Nature Methods, 7(9), 701-708. https://doi.org/10.1038/ nmeth.148 